\title{
Type-II Dirac cone and Dirac cone protected by nonsymmorphic symmetry in carbon-lithium compound $\left(\mathrm{C}_{4} \mathrm{Li}\right)$
}

\author{
Armindo S. Cuamba ${ }^{1}$, Pavan Hosur ${ }^{1}$, Hong-Yan Lu ${ }^{1,2}$, Lei Hao ${ }^{1,3}$, and C. S. Ting ${ }^{1}$ \\ 1 Texas Center for Superconductivity and Department of Physics, \\ University of Houston, Houston, Texas 77204, USA \\ ${ }^{2}$ School of Physics and Electronic Information, Huaibei Normal University, Huaibei 235000, China \\ 3 Department of Physics, Southeast University, Nanjing 210096, China
}

(Dated: August 29, 2018)

\begin{abstract}
In this work, we predict a novel band structure for Carbon-Lithium $\left(\mathrm{C}_{4} \mathrm{Li}\right)$ compound using the first-principles method. We show that it exhibits two Dirac points near the Fermi level; one located at $\mathrm{W}$ point originating from the nonsymmophic symmetry of the compound, and the other one behaves like a type-II Dirac cone with higher anisotropy along the $\Gamma$ to $\mathrm{X}$ line. The obtained Fermi surface sheets of the hole-pocket and the electron-pocket near the type-II Dirac cone are separated from each other, and they would touch each other when the Fermi level is doped to cross the type-II Dirac cone. The evolution of Fermi surface with doping is also discussed. The bands crossing from $\mathrm{T}$ to $\mathrm{W}$ make a line-node at the intersection of $\mathrm{k}_{x}=\pi$ and $\mathrm{k}_{y}=\pi$ mirror planes. The $\mathrm{C}_{4} \mathrm{Li}$ is a novel material with both nonsymmorphic protected Dirac cone and type-II Dirac cone near the Fermi level which may exhibit exceptional topological property for electronic applications.
\end{abstract}

PACS numbers:

Introduction: Carbon has been extensively investigated due to its exceptional property and easier syntheses in the related industry. Graphite $\frac{1}{\underline{1}}$ and diamond are some common forms of carbon found in nature. For instance, diamond is an insulator with strong elastic properties and has been used to achieve high-pressure environment ${ }^{2}$. Other carbon structures were predicted to be stable ${ }^{\frac{3}{}}$ and synthesized under different conditions. Graphene forms a Dirac cone at high symmetry point with zero density of states at the Fermi level. Intercalated graphene with alkaline metals such as lithium was reported to exhibit a metallic band structure and superconductivity ${ }^{4}$. In addition, carbon-lithium structure was used to generate lithium battery ${ }^{5}$.

The realization of carbon structure with nontrivial topology has become an interesting topic recently, and different structures were proposed. For example, the two-dimensional carbon structure; the $\alpha$-graphyne, $\beta$ graphyne, and $(6,6,12)$-graphyne exhibit Dirac cones ${ }^{3}$. The three-dimensional carbon structure; the bco- $\mathrm{C}_{16}{ }^{6}$, and bct- $\mathrm{C}_{16^{\frac{7}{2}}}$ were predicted to be a line-node semimetal protected by the mirror symmetry, and the graphene has type-I Dirac cone which is similar to the Dirac cone of $\mathrm{Na}_{3} \mathrm{~B}^{i}$ and $\mathrm{Cd}_{3} \mathrm{As}_{2}{ }^{\underline{9}}$ material. The nonsymmorphic ${ }^{10}$ material was predicted to exhibit a different type of Dirac cone characterized by having a crossing of two-fold degenerate bands and result in a four-fold point node on $\mathrm{HfI}_{3}$ compound 11 . This type of crossing is located at the high symmetry point in the Brillouin zone (BZ). Later the Angle-resolved photoemission experiments (ARPES) confirmed the existence of this type of Dirac cone in $\mathrm{ZrSiS}$ compound $\frac{12}{2}$. Besides, there exist other materials that are Dirac node-semimetals protected by nonsymmorphic symmetry $\stackrel{13}{ }$.

The Weyl semimetal material are classified as type$\mathrm{I}^{14-16}$ and type-II ${ }^{17,18}$ crossings and both were realized.
Similarly, the Dirac semimetal can also be classified as type-I and type-II. While many structures have been classified as type-I Dirac semimetals ${ }^{8,9}$, the search for type-II Dirac semimetals is still an interesting and emergent issue. Although some compounds were predicted to have type-II Dirac fermions ${ }^{19-22}$, and its existence has only been observed in $\mathrm{PtSe}_{2}{ }^{23}, \mathrm{PtTe}_{2}{ }^{24}$ and $\mathrm{PdTe}_{2}{ }^{25}$. Unfortunately, the observed Dirac cone ${ }^{23}-25$ is located away from the Fermi level that would make it be difficult to study the property pertinent to the type-II Dirac cone experimentally. Because of the existence of type-I Dirac cones at the Fermi level in graphene and its related materials $\frac{3.6 .7}{1}$, we speculate that these graphene-based compounds plus the simplest metallic element Li may generate the possible system to have the type-II Dirac cone near the Fermi level with relevant electronic structures.

In this work, using the first-principles method, various structures of graphene-based compounds with $\mathrm{Li}$ atoms were examined. We found that the band structure of the carbon-lithium compound $\left(\mathrm{C}_{4} \mathrm{Li}\right)$ (see Fig. 1) has two crossings; one located at $0.11 \mathrm{eV}$ above the chemical potential corresponding to a type-II Dirac cone, and the other crossing is located at $0.34 \mathrm{eV}$ above the chemical potential (see Fig.2). The latter crossing corresponds to a type-I Dirac cone at point $\mathrm{W}$ forming a node-line from $\mathrm{W}$ to $\mathrm{T}$ protected by the nonsymmorphic symmetry of the compound. If all the Li atoms are removed from this compound, the remaining carbon atoms would have the orthorhombic structure of bco- $\mathrm{C}_{16} \frac{6}{}$. The formation energy, phonon dispersion, Fermi surface and the corresponding density of states (DOS) are all calculated. The formation energy of $\mathrm{C}_{4} \mathrm{Li}$ is smaller than that of $\mathrm{C}_{2} \mathrm{Li}_{2}$ structure indicating that $\mathrm{C}_{4} \mathrm{Li}$ is thermodynamically stable. The phonon dispersion shows no imaginary frequency, and this implies that the structure is also dynamically 


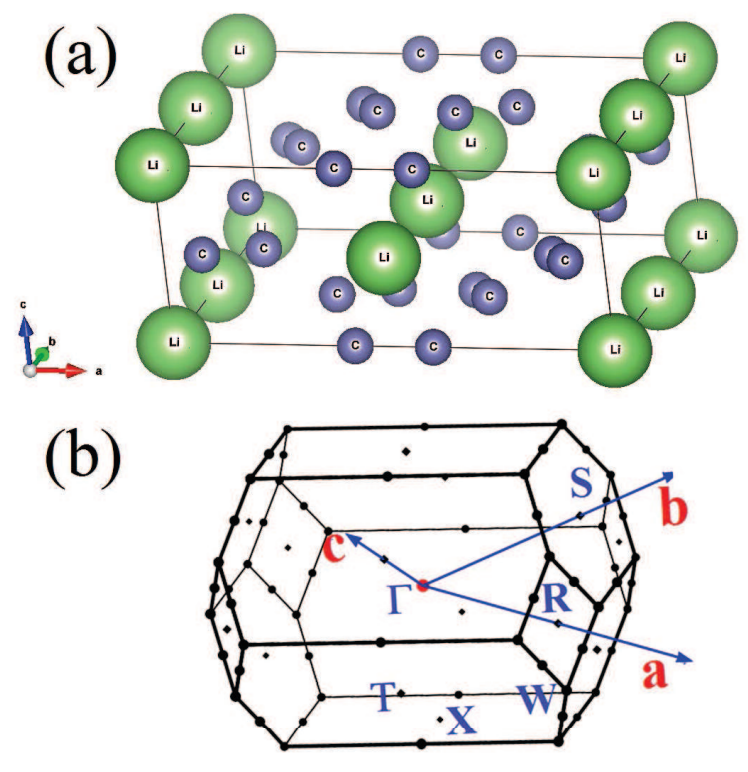

FIG. 1: (colour online) (a) Crystal structure of the CarbonLithium $\mathrm{C}_{4} \mathrm{Li}$. The carbon atoms form a tree-dimensional chain with honeycomb structure when viewed from (001) plane while the lithium atoms form a chain in the (010) plane, and (b) is the corresponding primitive Brillouin zone.

stable. The total DOS is mainly contributed from the bands crossing the Fermi level. The carbon atoms have more contribution to DOS while the lithium atoms have less contribution. Our x-rays diffraction (XRD) clearly show a peak characteristic for three-dimensional carbonbased structure and new peaks are found only in $\mathrm{C}_{4} \mathrm{Li}$.

Computational details, crystal structure and formation energy: The calculation of phonon frequencies is performed by using density functional perturbation theory (DFPT) which is implemented in quantum espresso package $\frac{26}{\underline{ }}$ using the pseudopotential of the Perdew-Burke-Ernzerhof (PBE) type with GGA exchange-correlation potential. The electronic structure calculation was performed by using VASP (Vienna $a b$ initio simulation package $) 27$. The input crystal parameters are the following: $\mathrm{a}=7.84262 \AA, \mathrm{b}=4.8872 \AA$, and $\mathrm{c}=3.8680 \AA$. The self-consistent calculation for the electronic structure is performed in the $8 \times 8 \times 8 \mathrm{k}$-grid, and the cut-off energy for the wave function is $380 \mathrm{Ry}$. The crystal structure and the XRD simulations were obtained by using the VESTA package ${ }^{29}$.

The $\mathrm{C}_{4} \mathrm{Li}$ compound crystallizes in the orthorhombic structure with space group Imma(74). This space group is characterized by having both nonsymmorphic and the inversion symmetry. The crystal structure of $\mathrm{C}_{4} \mathrm{Li}$ is illustrated in Fig.1. The structure is composed of 16 carbon atoms and 4 lithium atoms in the conventional unit cell. The carbon atoms form a graphene-like structure when viewed from (001) plane (the plane perpendicular to c axis), and the lithium atoms are located at the center of lattice making this structure a body-center-
TABLE I: Calculated parameters of different Carbon-Lithium structures by GGA method: The volume of the structure per atoms, bond distance $\mathrm{d}_{C-C}$ and $\mathrm{d}_{L i-L i}$, and the total formation energy per atom of $\mathrm{C}_{2} \mathrm{Li}_{2}(\mathrm{Immm})^{30}, \mathrm{C}_{2} \mathrm{Li}_{2}(\mathrm{Fm}-$ $3 m)^{30}, \mathrm{C}_{4} \mathrm{Li}($ Imma $), \mathrm{C}_{6} \operatorname{Li}(P 6 / m m m)^{4}, \mathrm{C}_{12} \operatorname{Li}(P 6 / m m m)^{31}$ and $\mathrm{C}_{18} \operatorname{Li}(P 6 / \mathrm{mmm})^{32}$

\begin{tabular}{ccccc}
\hline \hline Structure & $\begin{array}{c}\text { Volume } \\
\left(\AA^{3} / \text { atom }\right)\end{array}$ & $\begin{array}{c}\mathrm{d}_{C-C} \\
(\AA)\end{array}$ & $\begin{array}{c}\mathrm{d}_{\text {Li-Li }}(\AA) \\
(\mathrm{R}) / \text { atom })\end{array}$ & $\begin{array}{c}\mathrm{E}_{\text {total }} \\
(\mathrm{R})\end{array}$ \\
\hline \hline $\mathrm{C}_{2} \mathrm{Li}_{2}($ Immm $)$ & 7.160 & 1.2651 & 2.6647 & -0.334682 \\
$\mathrm{C}_{2} \mathrm{Li}_{2}(F m-3 m)$ & 17.686 & 4.2179 & 2.9825 & -0.169551 \\
$\mathrm{C}_{4} \operatorname{Li}($ Imma $)$ & 7.864 & 1.4575 & 2.4460 & -0.469653 \\
$\mathrm{C}_{6} \operatorname{Li}(P 6 / m m m)$ & 8.497 & 1.444 & 4.3426 & -0.550839 \\
$\mathrm{C}_{12} \operatorname{Li}(P 6 / m m m)$ & 8.655 & 1.442 & 4.3259 & -0.595709 \\
$\mathrm{C}_{18} \operatorname{Li}(P 6 / m m m)$ & 8.870 & 1.432 & 4.2971 & -0.612133 \\
\hline \hline
\end{tabular}

orthorhombic structure. The nearest-neighbor bond distance of the $\mathrm{Li}-\mathrm{C}$ is $2.0214 \AA$, the bonding distance of lithium atoms is $2.4460 \AA$, and the carbon bonding distance is $1.4575 \AA$. Table I shows the volume of the structure per atom, the bond distances $\left(\mathrm{d}_{C-C}\right.$ and $\left.\mathrm{d}_{L i-L i}\right)$ and the corresponding formation energy of different Carbon-Lithium structures which were synthesized and compared with the new structure $\mathrm{C}_{4} \mathrm{Li}$. The bonding distance of $C-C$ and $L i-L i$ for $\mathrm{C}_{4} \mathrm{Li}$ are within the limits of carbon-lithium structures. The total energy is -188.641 Ry and the formation energy per atom is negative (-0.484573 Ry) which suggest the thermal stability of the structure. Furthermore, the formation energy(FE) of $\mathrm{C}_{4} \mathrm{Li}$ is less than the $\mathrm{FE}$ of the known structure, the $\mathrm{C}_{2} \mathrm{Li}_{2}(\mathrm{Immm})^{30}$ and $\mathrm{C}_{2} \mathrm{Li}_{2}(\mathrm{Fm}-3 m)^{30}$ respectively, also indicating that $\mathrm{C}_{4} \mathrm{Li}$ is thermodynamically stable. The angle formed by the $\mathrm{Li}-\mathrm{C}-\mathrm{Li}$ is $139.049^{\circ}$, and the minimal angle formed by the C-Li-C is $40.951^{\circ}$.

Results and discussion: The electronic structures are calculated with spin-orbit coupling interaction(SOC). Since the structure have the inversion symmetry and time-reversal symmetry the bands structure should be degenerate everywhere in the BZ. The carbon and lithium both have week SOC, therefore, there is no significant change of the band structure with the inclusion of SOC. The band structure of $\mathrm{C}_{4} \mathrm{Li}$ calculated along the high symmetry lines in the first BZ is presented in Fig 2(a). The higher dispersive bands cross the Fermi level forming two different types of Dirac fermions. There is one crossing located at $\mathrm{W}(0.75,-0.25,-0.25)$ high symmetry point and the other crossing is located between $\Gamma$ to $\mathrm{X}(0.5,-0.5,-0.5)$ points in the first BZ. The first one shown in Fig 2(b) is originated from the crossing of the twofold-degenerate bands located from $\mathrm{W}$ to $\mathrm{R}$ and leads to formation of fourfold-degenerate band from $\mathrm{T}$ to $\mathrm{W}$ high symmetry line respectively which is different from other known Dirac crossing because it is located at 0.346 $\mathrm{eV}$ above the Fermi level. The bands located from $\mathrm{W}$ to $\mathrm{T}$ makes a line-node at the boundary of the Brillouin zone which coincides with the crossing of the mirror planes 


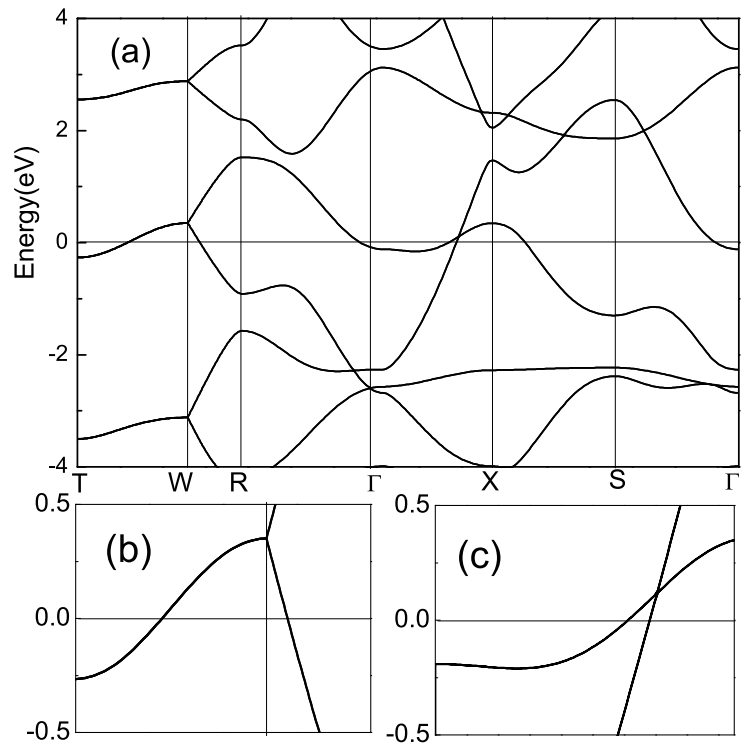

FIG. 2: (colour online) (a) Band structure of $\mathrm{C}_{4} \mathrm{Li}$ along high symmetry lines. (b) The line-node if formed from $\mathrm{T}$ to $\mathrm{W}$ located at the intersection of $\mathrm{k}_{x}=\pi$ and $\mathrm{k}_{y}=\pi$ mirror planes, and (c) the other crossing from $\Gamma$ to $\mathrm{X}$ is the type-II Dirac cone.
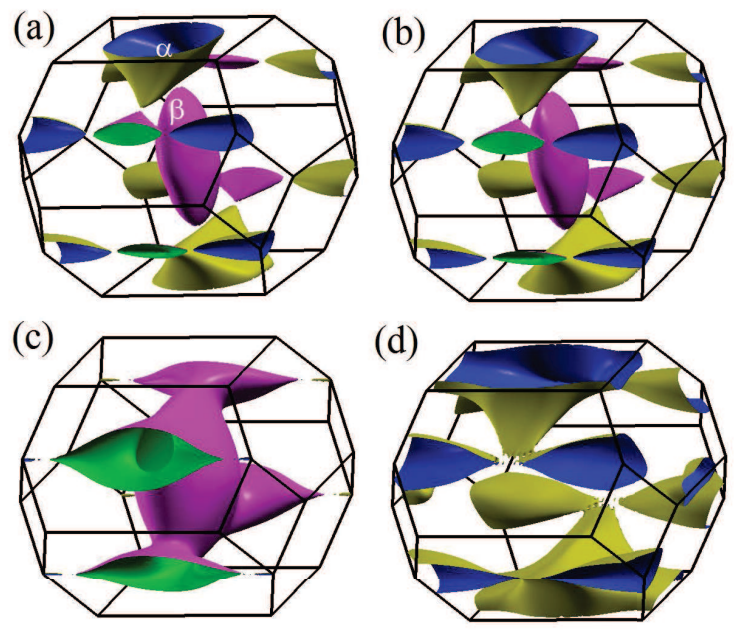

FIG. 3: (colour online) Fermi surfaces of $\mathrm{C}_{4} \mathrm{Li}$ obtained at different energy levels. The Fermi surface (a), (b), (c) and (d) are plotted at $0 \mathrm{eV}, 0.11 \mathrm{eV}, 0.40 \mathrm{eV}$ and $-0.19 \mathrm{eV}$, respectively.

$\mathrm{k}_{x}=\pi$ and $\mathrm{k}_{y}=\pi$ in the conventional cell. This can be easily seen in Fig. 4 and the symmetry analysis at higher symmetry points of the primitive cell discussed in the Supplementary Material ${ }^{33}$.

The second crossing is the so called type-II Dirac cone which is located at $0.11 \mathrm{eV}$ above the Fermi level as illustrated in Fig[2(c). The principal characteristic of this type of crossing appears to violate the Lorentz invariance, which is an important principle in Higher Energy Physics.
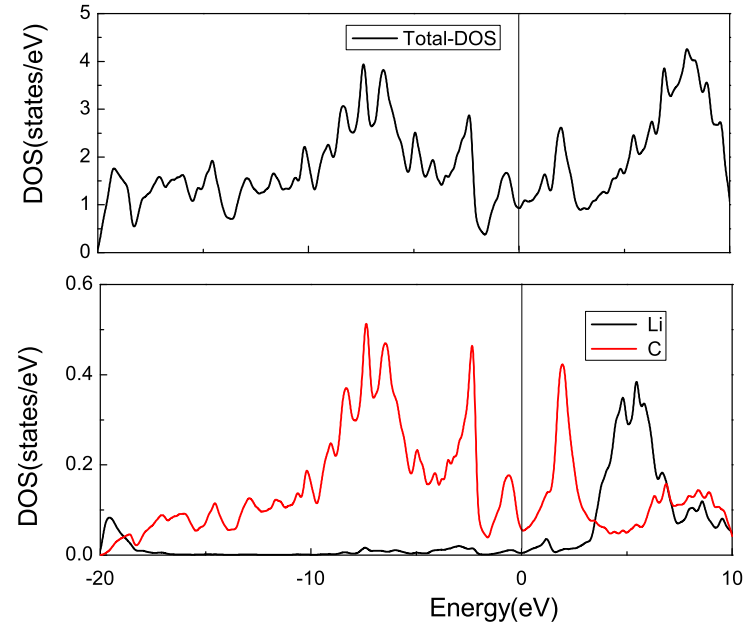

FIG. 4: (colour online) (a) The total electronic density of states of $\mathrm{C}_{4} \mathrm{Li}$ compound and (b) the partial density of states of carbon and lithium atoms.

The presence of the type-II Dirac semimetal suggests the existence of new physics that has never been explored before. The band structure of $\mathrm{C}_{4} \mathrm{Li}$ compound is the first carbon-based material to exhibit this type of electronic structure which is different from the type-I Dirac cone observed in graphene and in $\mathrm{Na}_{3} \mathrm{Bi}^{8}$ and $\mathrm{Cd}_{3} \mathrm{As}_{2}{ }^{9}$. The Dirac cone is titled along $\Gamma$ to $\mathrm{X}(0.5,-0.5,-0.5)$ high symmetry line and is anisotropic; the dispersion has different slopes in all direction. Along the principal diagonal of the unit cell, the anisotropy is enhanced, and this corresponds to the higher separation distance between the lithium atoms. The crossing is located at the special coordinate $(0.3333,-0.3333,-0.3333)$ along the $\Gamma$ to $\mathrm{X}$ where the hole-like and the electron-like pockets touch each other.

The Fermi surface of $\mathrm{C}_{4} \mathrm{Li}$ at different doping or chemical potential are shown in Fig, 3 . Fig 3(a) illustrates the Fermi surface at zero doping, which is composed of the electron pocket $\beta$, the hole pocket $\alpha$ constituting the characteristics of the type-II Dirac cone along the $\Gamma$ to $\mathrm{X}$ line, plus the other Fermi sheets corresponding to the band crossing from T-W-R high symmetry line. The two pockets $\beta$ and $\alpha$ would touch each other when the chemical potential crosses the Dirac node at the $0.11 \mathrm{eV}(\mathrm{Fig} \mathbf{3}(\mathrm{b}))$ if the compound is properly doped with electrons. The Fermi surfaces (c) and (d) in Fig 3 correspond to higher electron and hole-doping respectively. With heavy electron (hole) doping, there is the disappearance of the hole (electron) pocket.

The corresponding total and the partial DOS are presented in the Fig.4. The total DOS is enhanced at the Fermi level which is due to the nontrivial bands crossing the Fermi level. The carbon atoms have dominant contribution to the DOS, while the lithium atoms have less contribution. The Dirac cones in this structure should be easier studied by ARPES or magnetoresistance ex- 


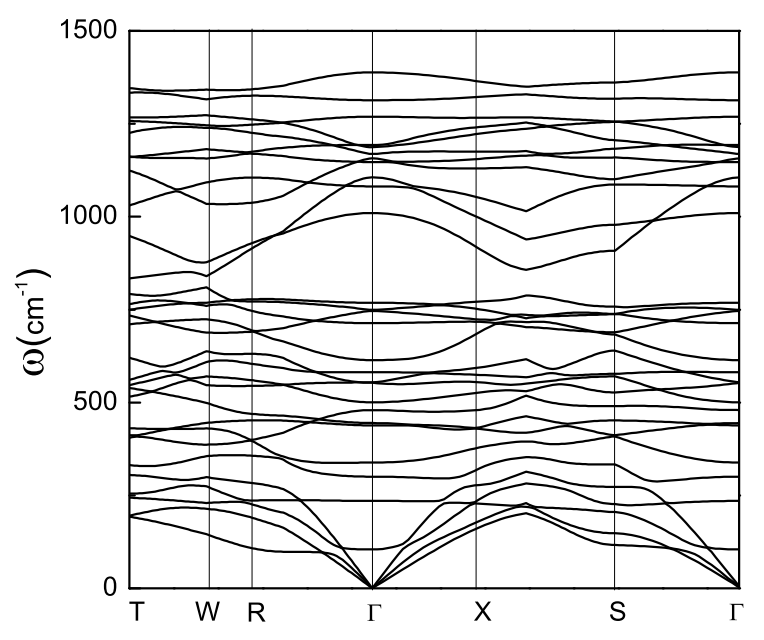

FIG. 5: (colour online) Phonon dispersion for $\mathrm{C}_{4} \mathrm{Li}$ structure.

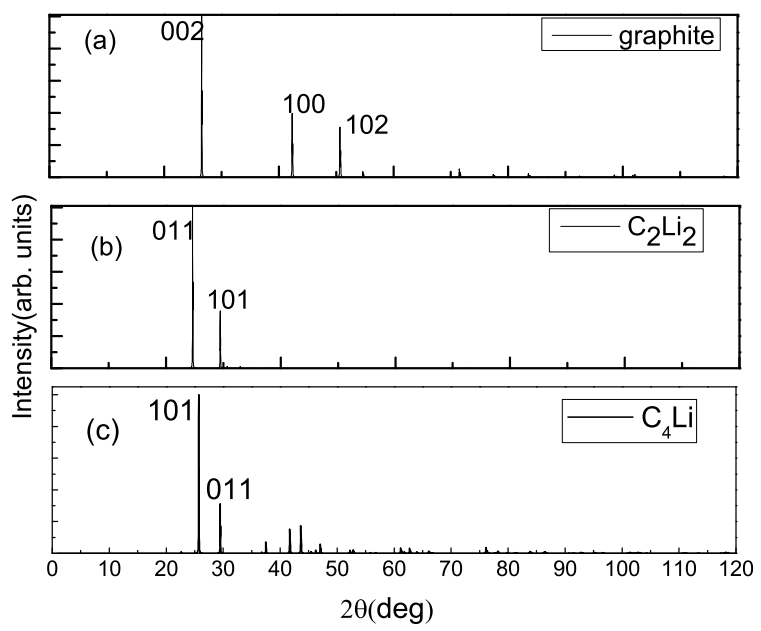

FIG. 6: (colour online) X-ray diffraction pattern (XRD) patterns of (a) graphite, (b) $\mathrm{C}_{2} \mathrm{Li}_{2}(\operatorname{Immm})$, and (c) $\mathrm{C}_{4} \mathrm{Li}$ taken at the wavelength of $1.54423 \AA$.

periments because all the band close to Fermi level are nontrivial, so the signal coming from the density of states will not have any interference. The phonon dispersion of the $\mathrm{C}_{4} \mathrm{Li}$ is presented Fig 5 . The spectra do not show any imaginary frequencies, indicating that the structure is dynamically stable.

In order to compare our theory with future experi- ments for the identification of $\mathrm{C}_{4} \mathrm{Li}$ compound, we perform X-ray diffraction simulation 29 pattern(XRD) at a wavelength of $1.54423 \AA$ and compare it with the known structure of Lithium-carbide, and the results are shown in Fig 6. The peak of $\mathrm{C}_{4} \mathrm{Li}$ at $2 \theta=25.69^{0}$ is also present in graphite and in $\mathrm{C}_{2} \mathrm{Li}_{2}(\mathrm{Immm})$ structure, while, the one at $29.60^{\circ}$ is only present in both $\mathrm{C}_{4} \mathrm{Li}$ and $\mathrm{C}_{2} \mathrm{Li}_{2}(\mathrm{Immm})$ structures. This indicates the existence of similar characteristic of carbon-lithium structure. There are new peaks above $30^{\circ}$ at $41.67^{\circ}$ and $43.60^{\circ}$ which are characteristics of $\mathrm{C}_{4} \mathrm{Li}$ and the experiments could look for these peaks in order to identify this compound.

Conclusion: We have predicted the new stable phase of the carbon-lithium $\left(\mathrm{C}_{4} \mathrm{Li}\right)$ by means of the first-principles calculation method. The obtained band structure shows two crossings, one located at $\mathrm{W}$ high symmetry point and the other one is the type-II Dirac cone located between the $\Gamma$ to $\mathrm{X}(0.5,-0.5,-0.5)$ points . The band crossing remains gapless with the inclusion of SOC due to small SOC strength of both carbon and lithium atom, and a line-node is formed at the intersection of the mirror planes at $\mathrm{k}_{x}=\pi$ and $\mathrm{k}_{y}=\pi$. Its formation energy is shown to be lower than that of the known structure of $\mathrm{C}_{2} \mathrm{Li}_{2}$ indicating the structure of $\mathrm{C}_{4} \mathrm{Li}$ is thermodynamically stable. Our phonon dispersion calculation also indicates the compound to be dynamically stable. Hopefully, the experimentalists will be able to synthesize this compound in the future, and to discover the first material that would have the type-II Dirac cone near the Fermi energy. We also expect that $\mathrm{C}_{4} \mathrm{Li}$ compound is going to exhibit exceptional topological property and to have the potential for electronic applications.

\section{Acknowledgments}

This work is supported by the Texas Center for Superconductivity at the University of Houston and the Robert A. Welch Foundation (Grant No. E-1146), the National Natural Science Foundation of China (Grant No. 11574108 ), the Natural Science Foundation of Anhui Province in China (Grant No. 1408085QA12), and the Natural Science Research Project of Higher Education Institutions of Anhui Province in China (Grant No. KJ2015A120). The numerical calculations were performed at the Center for Advanced Computing and Data at the University of Houston.
1 A. K. Geim and K. S. Novoselov, Nature Materials 6, 183 - 192 (2007).

2 Paul F. McMillin, Chem. Soc. Rev. 35, 855-857, (2006).

3 Daniel Malko, Christian Neiss, Francesc Vies, and Andreas Grling, Phys. Rev. Lett. 108, 086804 (2012).

${ }^{4}$ Gianni Profeta, Matteo Calandra, and Francesco Mauri, Nature Physics 8, 131134 (2012).
5 Jusef Hassoun, Francesco Bonaccorso, Marco Agostini, Marco Angelucci, Maria Grazia Betti, Roberto Cingolani, Mauro Gemmi, Carlo Mariani, Stefania Panero, Vittorio Pellegrini, and Bruno Scrosati, Nano Lett., 2014, 14 (8), pp 49014906 (2014).

6 Jian-Tao Wang, Hongming Weng, Simin Nie, Zhong Fang, Yoshiyuki Kawazoe, and Changfeng Chen, Phys. Rev. 
Lett. 116, 195501(2016).

7 Yong Cheng, Xing Feng, Xiaoting Cao, Bin Wen, Qian Wang, Yoshiyuki Kawazoe, Puru Jena, Small, 13, 1602894 (2017).

8 Z. K. Liu, B. Zhou, Y. Zhang, Z. J. Wang, H. M. Weng, D. Prabhakaran, S.K. Mo, Z. X. Shen, Z. Fang, X. Dai, Z. Hussain, Y. L. Chen, Science 343, Issue 6173, 864-867 (2014).

9 Z. K. Liu, J. Jiang, B. Zhou, Z. J. Wang, Y. Zhang, H. M. Weng, D. Prabhakaran, S-K. Mo, H. Peng, P. Dudin, T. Kim, M. Hoesch, Z. Fang, X. Dai, Z. X. Shen, D. L. Feng, Z. Hussain Y. L. Chen, Nature Materials 13, 677681 (2014).

10 S. A. Parameswaran, A. M Turner, D. P. Arovas, and A. Vishwanath, Nature Phys. 9, 299303 (2013).

11 Q. D. Gibson, L. M. Schoop, L. Muechler, L. S. Xie, M. Hirschberger, N. P. Ong, R. Car, and R. J. Cava Phys. Rev. B 91, 205128 (2015).

12 Leslie M. Schoop, Mazhar N. Ali, Carola Straer, Andreas Topp, Andrei Varykhalov, Dmitry Marchenko, Viola Duppel, Stuart S. P. Parkin, Bettina V. Lotsch and Christian R. Ast, Nature Communications 7, 11696 (2016).

13 C. Chen, X. Xu, J. Jiang, S.-C. Wu, Y. P. Qi, L. X. Yang, M. X. Wang, Y. Sun, N. B. M. Schrter, H. F. Yang, L. M. Schoop, Y. Y. Lv, J. Zhou, Y. B. Chen, S. H. Yao, M. H. Lu, Y. F. Chen, C. Felser, B. H. Yan, Z. K. Liu, and Y. L. Chen, Phys. Rev. B 95, (2017).

${ }^{14}$ L. X. Yang, Z. K. Liu, Y. Sun, H. Peng, H. F. Yang, T. Zhang, B. Zhou, Y. Zhang, Y. F. Guo, M. Rahn, D. Prabhakaran, Z. Hussain, S.-K. Mo, C. Felser, B. Yan Y. L. Chen, Nature Physics 11, 728732 (2015).

15 Xu N, Weng HM, Lv BQ, Matt CE, Park J, Bisti F, Strocov VN, Gawryluk D, Pomjakushina E, Conder K, Plumb NC, Radovic M, Auts G, Yazyev OV, Fang Z, Dai X, Qian T, Mesot J, Ding H, Shi M. Nat Commun, 11006, (2017).

16 Yan Sun, Shu-Chun Wu, and Binghai Yan, Phys. Rev. B 92, 115428 (2015).

17 Ke Deng, Guoliang Wan, Peng Deng, Kenan Zhang, Shijie Ding, Eryin Wang, Mingzhe Yan, Huaqing Huang, Hongyun Zhang, Zhilin Xu, Jonathan Denlinger, Alexei Fedorov, Haitao Yang, Wenhui Duan, Hong Yao, Yang Wu, Shoushan Fan, Haijun Zhang, Xi Chen Shuyun Zhou, Nature Physics 12, 11051110 (2016).
18 Alexey A. Soluyanov, Dominik Gresch, Zhijun Wang, QuanSheng Wu, Matthias Troyer, Xi Dai B. Andrei Bernevig, Nature 527, 495498 (2015).

19 Tay-Rong Chang, Su-Yang Xu, Daniel S. Sanchez, ShinMing Huang, Guoqing Chang, Chuang-Han Hsu, Guang Bian, Ilya Belopolski, Zhi-Ming Yu, Xicheng Xu, Cheng Xiang, Shengyuan A. Yang, Titus Neupert, Horng-Tay Jeng, Hsin Lin, M. Zahid Hasan, arXiv:1606.07555

20 P-J Guo, H-C Yang, K. Liu, and Z. Yi Lu, Phys. Rev. B 95, 155112 (2017).

21 C. Le, S. Qin, X. Wu, X. Dai, P. Fu and J.g Hu, arXiv: 1606.05042

22 H. Huang, S. Zhou, and W. Duan, Phys. Rev. B 94, 121117 (2016).

${ }^{23}$ K. Zhang, M. Yan, H. Zhang, H. Huang, M. Arita, Z. Sun, W. Duan, Y. Wu, S. Zhou, arXiv:1703.04242

24 Mingzhe Yan, Huaqing Huang, Kenan Zhang, Eryin Wang, Wei Yao, Ke Deng, Guoliang Wan, Hongyun Zhang, Masashi Arita, Haitao Yang, Zhe Sun, Hong Yao, Yang Wu, Shoushan Fan, Wenhui Duan, Shuyun Zhou, arXiv:1607.03643

25 Han-Jin Noh, Jinwon Jeong, En-Jin Cho, Kyoo Kim, B.I. Min, and Byeong-Gyu Park Phys. Rev. Lett. 119, 016401 (2017).

26 P. Giannozzi, S. Baroni, N. Bonini, M. Calandra, and R. Car et. al, J. Phys. Condens. Matter 21395502 (2009).

27 G. Kresse and J. Furthmller, Phys. Rev. 5411169 (1996).

${ }^{28}$ Qi-Feng Liang, Jian Zhou, Rui Yu, Zhi Wang, and Hongming Weng, Phys. Rev. B 93, 085427 (2016).

${ }^{29}$ K. Momma and F. Izumi, J. Appl. Crystallogr, 44, 12721276 (2011).

30 Ruschewitz, U. Poettgen, R. Zeitschrift fuer Anorganische und Allgemeine, Chemie, 625, 1599-1603 (1999).

31 V. Avdeev, A. Savchenkova, L. Monyakina, I. Nikolskaya, and A. Khvostov, J. Phys. Chem. Sol. 57, 947 (1996).

32 N. A. W. Holzwarth, Steven G. Louie, and Sohrab Rabii, Phys. Rev. B 28, 1013 (1983).

33 Armindo S. Cuamba, Pavan Husor, Hong-Yan Lu, Lei Hao, and C. S. Ting, Supplementary Material: Type-II Dirac cone and Dirac cone protected by nonsymmorphic symmetry in carbon-lithium compound $\left(\mathrm{C}_{4} \mathrm{Li}\right)$. 